\title{
Complete flow conditioning gauzes
}

\author{
J. V. Taylor ${ }^{1}$
}

Received: 26 October 2018 / Revised: 10 January 2019 / Accepted: 13 January 2019 / Published online: 2 February 2019 (c) The Author(s) 2019

\begin{abstract}
This paper presents a novel method that can completely condition the flow into a turbomachinery experiment. A single, thick, 3D-printed gauze can be tailored to provide an exact stagnation pressure profile, flow angle distribution and turbulence intensity. The new method is superior to existing techniques as it provides accurate and cheap flow conditioning in just one component. It removes the requirement for separate endwall boundary layer generators, inlet guide vanes and turbulence grids. The paper is presented in two parts: first, the methods for designing complete flow conditioning gauzes are presented. Both 1D correlations and 3D CFD are used to design the vanes which form the gauze. The exit angle of the individual vanes is used to determine the whirl angle of the flow. Thickness is used to determine the mixing loss and thus achieve the pressure distribution and turbulence intensity. In the second part, two gauzes are designed and manufactured for two compressor testing applications. The first design is used to simulate a multi-stage embedded profile. This means that engine realistic flow conditions can be set up in a single stage compressor rig. The second design simulates the hub side stagnation pressure deficit that occurs at inlet to a core compressor downstream of an aero engine fan. Both applications demonstrate the fine control that can be achieved in an experiment using these gauzes.
\end{abstract}

\section{Graphical abstract}

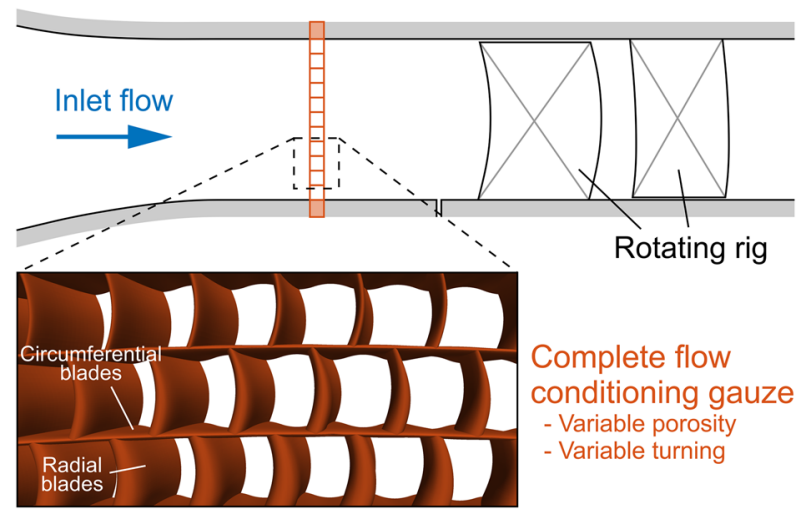

\section{Introduction}

The inlet flow to a rotating rig or wind tunnel is extremely important in determining the behaviour of the flow in the experiment itself. Traditional techniques use combinations

J. V. Taylor

jvt24@cam.ac.uk

1 Whittle Laboratory, Department of Engineering, University of Cambridge, Cambridge, UK of inlet guide vanes, endwall boundary layer generators and turbulence grids to condition the inlet flow. These methods can be relatively difficult to tune to the desired conditions and they only have coarse control over the flowfield. For example, endwall boundary layer generators can broadly increase thickness but they cannot tailor an exact boundary layer profile. Likewise, inlet guide vanes could be designed to vary whirl angle from hub to casing but they cannot replicate the local whirl found in a skewed endwall boundary layer. 


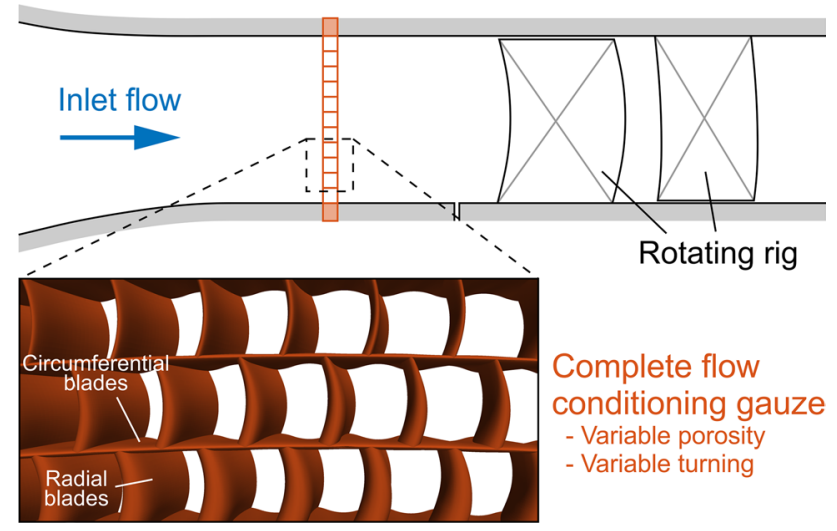

Fig. 1 Installation of a complete flow conditioning gauze in a rotating rig

This paper presents a new method for conditioning the inlet flow. With the advance of additive manufacturing techniques it is now possible to design a detailed gauze that can completely condition the flow by providing an exact stagnation pressure profile, flow angle distribution and turbulence intensity. Figure 1 shows an example of the gauze installed at the inlet to a rotating rig. The gauze is made up of both radial and circumferential blades. By tailoring the thickness and camber distributions of these blades the target flow condition can be achieved. Increasing thickness reduces gauze porosity, this results in increased mixing losses downstream and reduced stagnation pressure. Increasing the exit angle of the radial blades increases turning through the gauze, this results in a greater whirl angle of the flow.

Attempts to provide fine flow conditioning have been made in previous works. In Gunn and Hall (2014) a variable porosity gauze is used to replicate an inlet condition that would be seen by a boundary layer ingesting fan. In their work correlations from Livesey and Laws (1973) are used to set the porosity of the gauze to provide the required velocity profile. The gauze was 3D-printed in plastic with a fine resolution of holes, this produced a relatively smooth flow field. No attempt was made to replicate the angle distribution, caused by secondary flows, that would also be present in this application.

Work by Guimarães et al. (2018) focuses on secondary flows. In their flow conditioning device discrete blades are shaped to turn the flow to replicate the vortices that are seen in the ducts of military fans. However, blade resolution is relatively coarse, so loadings are high and wakes from the flow conditioning blades are still present at the inlet to the experiment.

This paper shows how to design a fine resolution gauze to provide a smooth flow of any profile. Two example applications are presented: first, the design of a gauze to simulate a multi-stage embedded compressor profile. Second, a design to simulate the hub side stagnation pressure deficit that occurs at inlet to a core compressor downstream of an aero engine fan.

\section{Gauze design}

This section presents the methods to design a complete flow conditioning gauze. This is an iterative process that determines the correct gauze porosity and exit angle to achieve a target stagnation pressure and whirl angle distribution. First, 1D correlations are used to initialise the design of the gauze. Second, 3D CFD is used to iterate the design until the target distribution is achieved. The meshing and CFD methods are covered alongside the detailed 3D aerodynamics in the passages of the gauze.

\subsection{D design}

The method to initialise a gauze design using correlations is presented in this part of the paper. To begin the process, the target stagnation pressure coefficient and whirl angle profiles are required. Figure 2 shows the profiles for the two applications presented in this paper. Second, the axial velocity profile upstream of the gauze must be measured or estimated. The dynamic head of the flow through each passage of the gauze can then be calculated along with the required pressure drop to achieve the target pressure profile. The correlation from Roach (1987) between pressure drop coefficient $\left(\Delta C_{\mathrm{Po}}\right)$ and porosity $(\beta)$ shown in Eq. (1), is used to fix the porosity distribution of the grid.

$\beta=\left(\frac{1}{\left(\frac{\Delta C_{\mathrm{Po}_{\mathrm{p}}}}{a}\right)^{\frac{1}{b}}+1}\right)^{\frac{1}{2}}$,

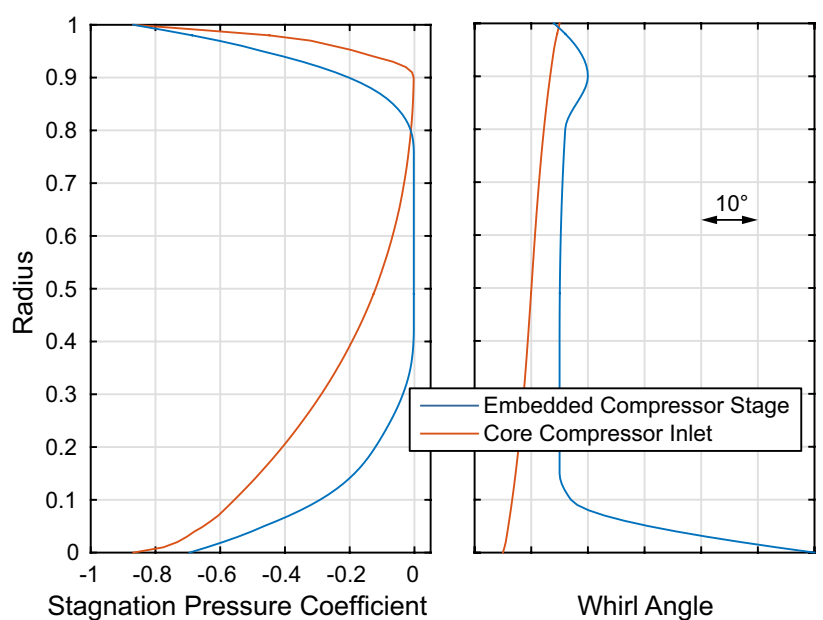

Fig. 2 Example target gauze exit profiles 
where $\Delta C_{\mathrm{Po}}$ is the drop in stagnation pressure normalised by the inlet dynamic head. In Roach (1987) $a$ and $b$, vary with Mach number, Reynolds number and grid geometry. The gauzes in this paper are more similar to round bar than square bar grids since the blades used have smooth leading edges, and therefore, low leading edge loss. Good results are obtained in this work using the round bar values $a=0.52$ and $b=1.0$. Similarly, when the gauzes are well designed the flow remains attached to the trailing edge. Therefore, the porosity is taken as the ratio of open area at the gauze trailing edge normalised by the total area of the passage.

It is possible to achieve the shape of the desired pressure profile with any number of gauzes which vary in their mean porosity. A reduced porosity design could be chosen if greater turbulence intensity was desired in the experiment. However, the stagnation pressure loss over the gauze would be increased and thus greater power is required from the wind tunnel or rotating rig to overcome these losses. The gauzes in this paper are to be tested on a medium speed, Mach $=0.4$, single stage compressor rig, so the pressure drop is selected to be as low as possible, therefore, the gauzes have a high porosity. The maximum porosity is determined by the structural limits of the gauze material. The gauzes in this paper were 3D-printed in UV-cured resin and so a minimum trailing edge thickness of $0.45 \mathrm{~mm}$ was chosen. This corresponds to a maximum porosity of 0.867 .

Once the porosity is fixed, the exit angle of the gauze $\left(\chi_{\mathrm{TE}}\right)$ can be calculated. It assumed that inviscid and viscous deviation within the passage of the gauze is low so the whirl is equal to the blade exit angle at the trailing edge plane. However, the whirl angle of the flow $\left(\alpha_{\text {mix }}\right)$ downstream of the gauze after it has mixed out will be significantly greater than at the gauze trailing edge plane. This can be calculated by applying continuity and conservation of momentum. The increase in whirl depends upon the porosity profile of the gauze as shown in Eq. (2).

$\tan \left(\chi_{\mathrm{TE}}\right)=\beta \times \tan \left(\alpha_{\text {mix }}\right)$

\subsection{Blading}

Blading the gauze is a trade off between structural integrity and smoothness of the resulting flow field. A large number of small passages would give a flow which is very smooth as the wakes from individual blades in the gauze would mix out before entering the experiment. However, the blades will become very thin, making it harder to resolve the features with a 3D-printer of limited resolution. The gauze is also more easily broken during cleaning, fitting or running.

The compressor rig used as an example in this paper has a midspan radius of $337 \mathrm{~mm}$ and a span of $55 \mathrm{~mm}$. The final gauze design chosen has 9 passages from hub to casing and 360 passages around the annulus. This gives a good balance between smoothness and structural integrity. The resulting passages are approximately $6 \mathrm{~mm}^{2}$. The trailing edge thickness of the circumferential blades is set to be $0.45 \mathrm{~mm}$ and is uniform across the span. The trailing edge thickness of the radial blades varies with the calculated porosity.

The selection of maximum thickness is also a trade, this time between structural integrity and pressure loss. If the blades are too thick, diffusion becomes high aft of maximum thickness. This can cause separations in the passages and increased loss and deviation. It is desirable to prevent separation as this introduces an uncertainty into the prediction of the resulting flow. A ratio of maximum thickness to trailing edge thickness of 1.7 was selected to balance these demands. Maximum thickness is located at 0.25 chord to further reduce the rate of diffusion.

The circumferential blades have a symmetric profile and form bands running around the annulus. The radial blades are cambered to provide the flow turning and vary across the span. Strong secondary flows can develop in the passages, this is because the aspect ratio of the blades is low and diffusion is high due to thickness-chord ratios of $20 \%$ or more. A circular arc camber line was chosen to relieve this problem as it pushes loading rearwards. Neighbouring rows of radial blades are offset by half a pitch as shown in Fig. 1 . This increases the rigidity of the gauze and mixing of the wakes shed from the radial blades.

\subsection{CFD method}

3D, steady RANS CFD was used to predict the flow in the gauze passages and the mixing downstream. The structured multi-block solver TURBOSTREAM was used, it is described in detail and validated in Brandvik and Pullan (2010). 120,000 cells were used per gauze passage and wall functions were used with $y^{+}$less than 3 on all walls. The Spalart-Allmaras turbulence model was used Spalart and Allmaras (1992). The gauze mesh was coupled to a mesh of the compressor rig with a mixing plane to ensure that the correct static pressure boundary condition was applied to the gauze exit. Further details of the mesh used in the test compressor and its geometry is given in Taylor and Miller (2016).

A novel blocking topology was required to run the gauzes in a structured CFD code. A mesh normally used to model a single passage calculation of a turbomachine requires periodic boundary conditions on the tangential faces. The mesh used to model a single pitch of this gauze requires periodic patches on the tangential, radial and axial faces to cope with the fact the passages are alternately offset in the tangential direction.

Figure 3 shows views of the blocks in an example passage, the meshing process was scripted in NUMECA's IGG. 7 blocks are required per passage in addition to the shared outlet block, this results in 64 blocks for the gauzes designed 


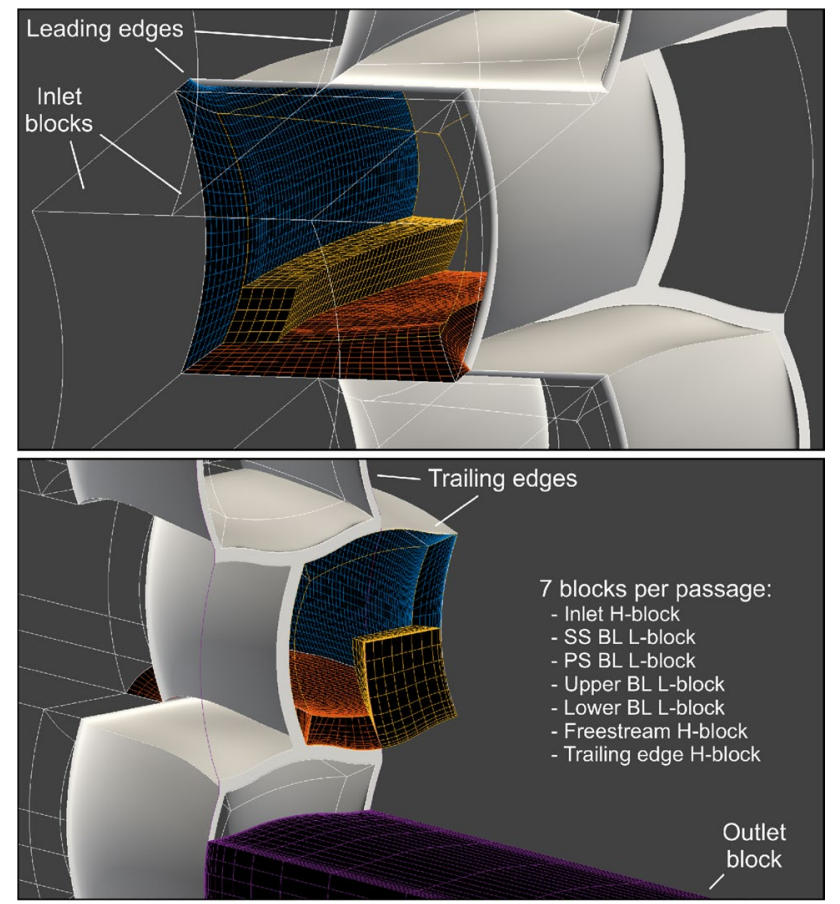

Fig. 3 Structured mesh topology

in this paper. 4 L-blocks model the boundary layer flow on the blade surfaces, these are wrapped around the smooth leading edges of both the radial and circumferential blades. 3 further blocks are required to complete each passage: the inlet, the freestream area and the trailing edge region. The outlet block joins all nine passages across the span and covers the blunt trailing edges of both the circumferential and radial blades.

\subsection{D design}

CFD is used to finalise the design of the gauze and investigate $3 \mathrm{D}$ flow features and blading options. The porosity and exit angle profiles that were initialised using the 1D correlations are used to blade the gauze and the first iteration is converged in CFD. The porosity and exit angles are iterated upon until the exit flow condition matches the target profile requested. This usually takes 3-4 iterations to converge.

Differences in flow angle are updated with changes to the exit angle in a 1:1 ratio. This is a stable scheme as it will always fall short of the target due to increased deviation. Differences in pressure are updated by calculating a pressure loss coefficient correction, a porosity correction can then be calculated using Eq. (1). A limit is placed upon the maximum porosity to ensure that the minimum trailing edge thickness is always met. Smoothing is applied before running the next geometry in CFD to ensure the gauze design does not vary too rapidly across the span.
Although the gauze accelerates the flow as it increases swirl the local diffusion in the passages can be high. This is due to overspeed caused by large thickness-chord and thickness-pitch ratios. Open corner separations that would normally only be observed on 2D compressor blades can occur. These cause significant loss and introduce uncertainty into the CFD prediction of the flow. By leaning the radial blades the open corner separations can be prevented and the losses reduced (Taylor and Miller 2016). To investigate this effect several gauze designs with differing values of tangential lean were generated and the results are plotted in Fig. 4. A tangential lean ratio of 0.12 was selected as it gives the optimum performance.

A complete flow conditioning gauze has a large wetted area and this results in a high loss coefficient that must be paid for with extra power from the rig or wind tunnel. To reduce the loss further the axial chord of the blades could be reduced. Figure 5 shows the effect of changing pitch-chord ratio on the gauze loss. CFD predicts the optimum value to be around 1.3, however, CFD predictions of optimum pitch-chord ratio have a large degree of uncertainty (Taylor 2015). For this work a pitch-chord ratio of 0.7 was chosen, this is a conservative choice that is closer to compressor

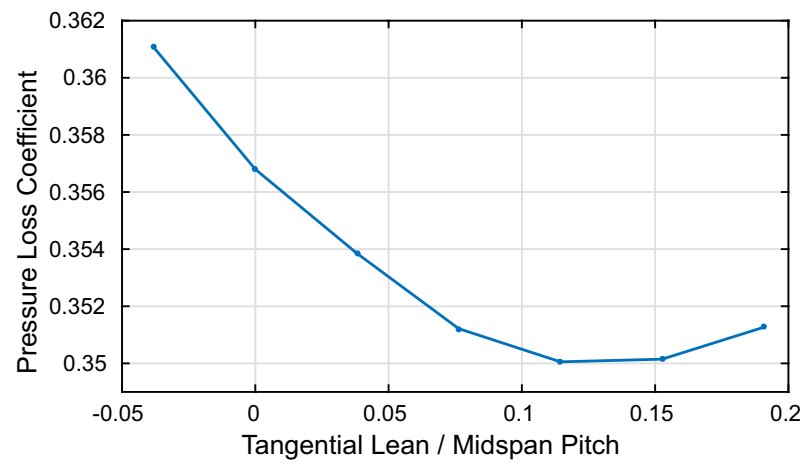

Fig. 4 Dependence of total loss on tangential lean computed with CFD

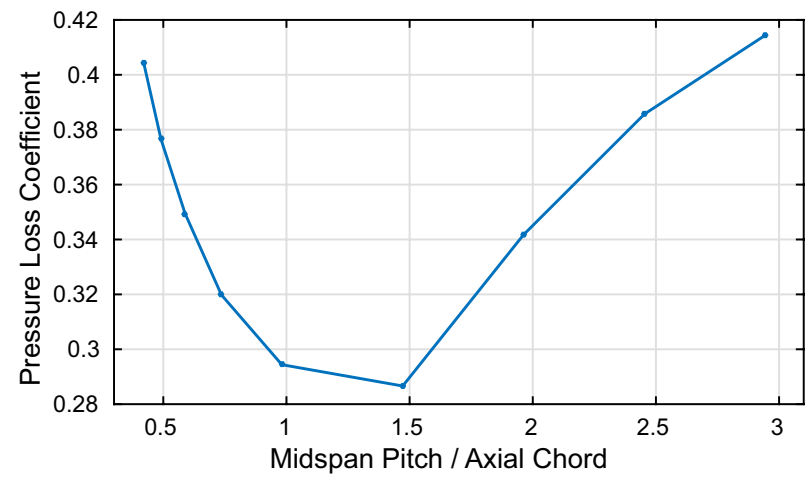

Fig. 5 Dependence of total loss on axial chord computed with CFD 
blading to ensure that the gauze is fully attached and thus the prediction of the developed profile is accurate.

For the applications presented in this paper the inlet conditions are required to be as smooth as possible. The wakes from the individual blades should, therefore, mix as quickly as possible. By offsetting the radial blades alternately in the tangential direction a single discrete radial wake is avoided. However, the circumferential blades will likely shed eight annular wakes into the experiment. This kind of flow would not be observed in a real turbomachine so the rate of mixing is increased downstream of the gauze by crenelating the trailing edges.

Figure 6 shows the predicted pressure profile downstream of gauzes with different magnitudes of crenelation. The magnitude of crenelation is specified by the ratio of displacement, $d$ to span, $h$. Whichever way the circumferential blades are perturbed should increase the rate of mixing as explained in McCormick and Bennett (1994). However, an increase in the smoothness of the pressure profile is only seen when the circumferential trailing edges are perturbed in the negative direction. This is due to increased trailing edge area and additional pressure losses in the corner regions if the gauze is perturbed in the positive direction. The only downside to perturbing in the negative direction is that deviation rises due to a reduction in the span of the radial blades. In this work it is chosen to perturb the circumferential blades by $-0.08 \mathrm{~d} / \mathrm{h}$.

\section{Gauze applications}

There are numerous potential uses for complete flow conditioning gauzes. These include setting up distorted inlet flows into rotating fan and turbine rigs, or flow conditioning in
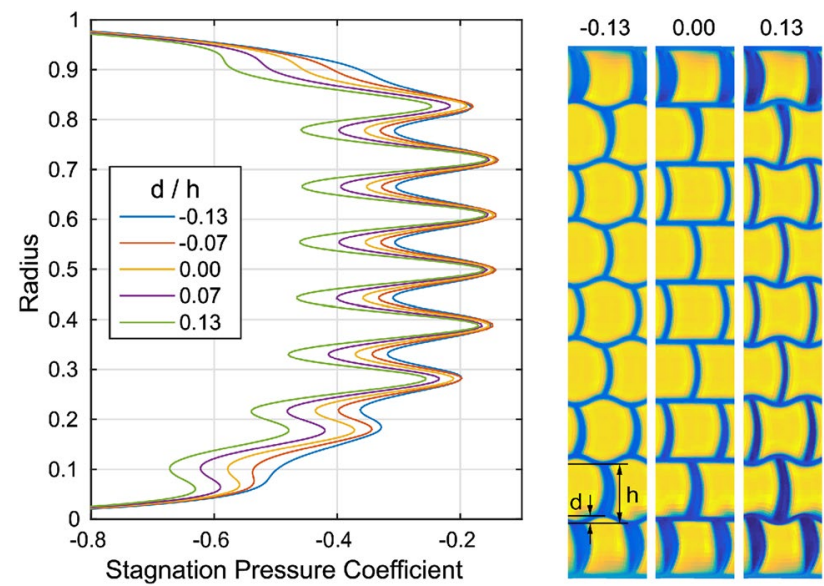

Fig. 6 Left: CFD profiles extracted eight axial chords downstream of trailing edge. Right: contours of stagnation pressure coefficient 0.15 axial chords downstream of trailing edge both low and high speed wind tunnels. This paper focusses on two compressor testing applications. First, the profile built up over many stages in a compressor is simulated. By creating a gauze to generate this profile, a single stage compressor rig can be used to realistically model the flow embedded in a multi-stage machine. Second, the hub side pressure deficit observed at the inlet to a core compressor is simulated. The fan root often causes high loss and when coupled with an engine side stator and a transition duct the first rotor in the core compressor sees a highly distorted profile. With this gauze design the effect of distortion on compressor performance can be realistically investigated.

\subsection{Manufacture}

Additive manufacture is the only realistic method of creating complete flow conditioning gauzes. It is only through the development of this technology that these flow conditioning devices have become possible. Polymer gauzes could be manufactured using SLA (stereolithography apparatus), SLS (selective laser sintering) or FDM (fused deposition modelling) and metal gauzes using DMLS (direct metal laser sintering) or SLM (selective laser melting).

Prototype parts were made in SLA and FDM, but a polyjet stereolithography process was finally selected for the experimental tests as it gave a good balance of print time, cost, rigidity and accuracy. A photo of the gauze installed in the rig inlet is shown in Fig. 7. It was printed in 24 sectors to allow easy assembly and to prevent distortion with heat and moisture.

The printer has an accuracy of $40 \mu \mathrm{m}$ in the radial and tangential directions and the gauze was built in $30 \mu \mathrm{m}$ layers from trailing edge up. Calibration of the thickness was required with prototypes, as any error in gauze thickness corresponds to an error in whirl angle. The gauze is supported at both the hub and casing to keep deflections due to pressure loading small. This was found to be particularly important as even small increases in flow temperature can cause a total loss of stiffness.

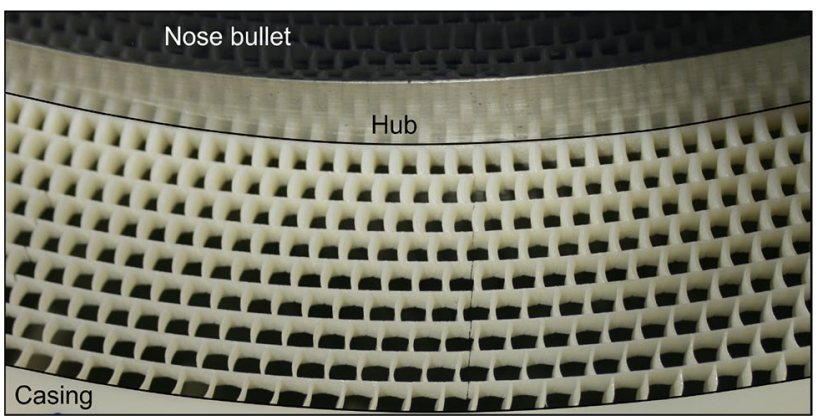

Fig. 7 Upstream view of polymer gauze installed in rig 


\subsection{Embedded stage profile}

The target profile shown in Fig. 2 is taken from the exit of a stator in a multi-stage compressor. It has thick endwall boundary layers on both the hub and casing. The upstream stator is unshrouded, therefore, the hub boundary layer has a large amount of positive skew. It was not possible to replicate this skew exactly and a limit of $45^{\circ}$ had to be imposed on the maximum exit angle of the blades. It was found that the gauze was unable to produce turning greater than this because of the high loading in the passage closest to the hub.

The results of the CFD design and experimental test are shown in Fig. 8. These results are taken from area traverses at the rotor inlet plane, 15 gauze axial chords downstream of the gauze trailing edge. A five hole probe was used to survey the flowfield, the diameter of its head is $1.9 \%$ of the span of the compressor. It is able to measure the stagnation pressure coefficient to an accuracy of $\pm 1 \%$ and whirl angle to $\pm 1^{\circ}$. For these area traverses 1026 sample points were used, 27 endwall clustered in the spanwise direction and 38 linearly spaced in the pitchwise direction.

It can be seen that the gauze produces the correct flow turning to within $1^{\circ}-2^{\circ}$ except close to the endwalls. Here the skew is broadly captured but has likely mixed out faster

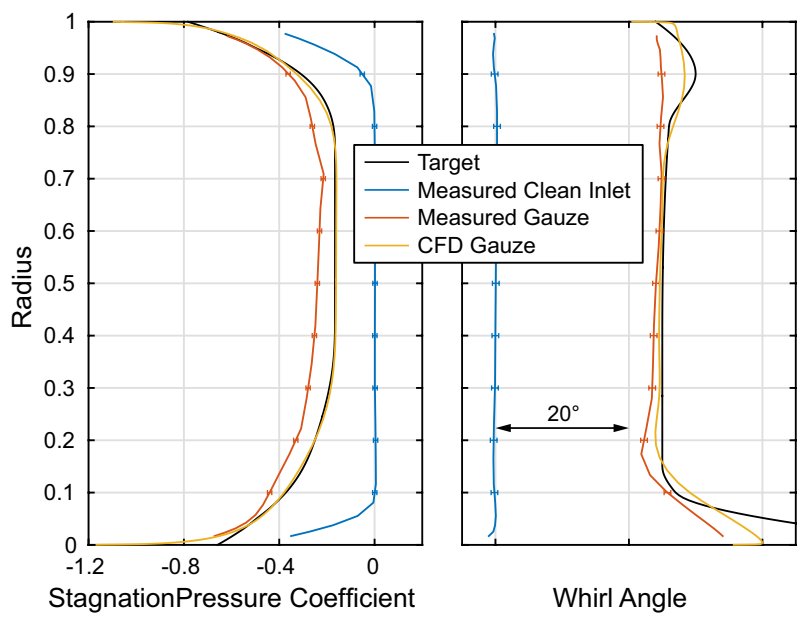

Fig. 8 CFD design and experimental test of embedded compressor stage gauze in the experiment than predicted with the CFD. The shape of the pressure profile has been reproduced accurately. However, the absolute loss is $23 \%$ greater than that predicted by the CFD. The most likely source of this error is the tolerances in the printing process. A discrepancy in loss of this magnitude corresponds to an error in trailing edge thickness of only $0.1 \mathrm{~mm}$. This increase in loss can be overcome with increased air power from the rig. Overall, the design and manufacturing processes are robust as the shape of the flow angle and pressure profiles are accurately reproduced.

Figure 9 shows an area traverse taken with the five hole probe at the rotor inlet plane over a $15^{\circ}$ sector. The flow is smooth with no discrete wakes from the radial or circumferential blades measurable. The join between two sectors of gauze causes only minor disturbance to the flow. The standard deviation in the pitchwise direction is 0.015 for stagnation pressure coefficient and $0.44^{\circ}$ for whirl angle. The measured flowfield is smoother than that predicted by the CFD, this indicates that the rate of mixing is higher in the experiment, likely due to wake shedding.

The use of a complete flow conditioning gauze in this application of represents a step-change improvement in accuracy and uniformity over the traditional combination of IGVs and endwall boundary layer generators. It is now possible to replicate the flow field embedded in a compressor spool in a single stage rig.

\subsection{Core compressor profile}

The target profile shown in Fig. 2 is taken upstream of the rotor row in the first stage an aero engine core compressor. It has a large stagnation pressure deficit originating at the hub and extending across $90 \%$ of the span. The target whirl angle profile varies smoothly from hub to tip as it would have been set by a variable inlet guide vane in the engine. The results of the CFD design and experimental test are shown in Fig. 10.

The shape of the pressure profile is replicated exactly, however once again the absolute loss $26 \%$ greater than the CFD prediction. The whirl angle distribution is correct to $1^{\circ}$ apart from at the endwalls where greater swirl is observed in the experiment. This suggests that the upstream axial velocity profile assumed in the design process is incorrect.
Fig. 9 Area traverse of embedded compressor stage gauze, 15 axial chords downstream of gauze trailing edge
Stagnation Pressure Coefficient

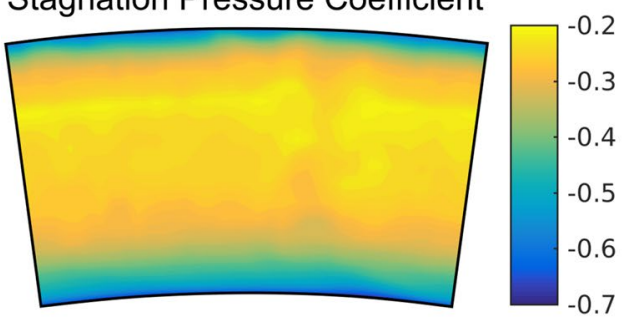

Whirl Angle / ${ }^{\circ}$

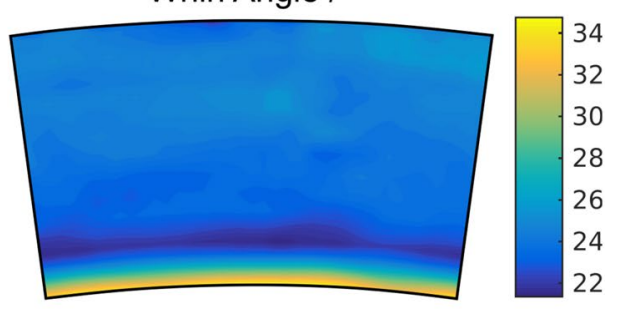




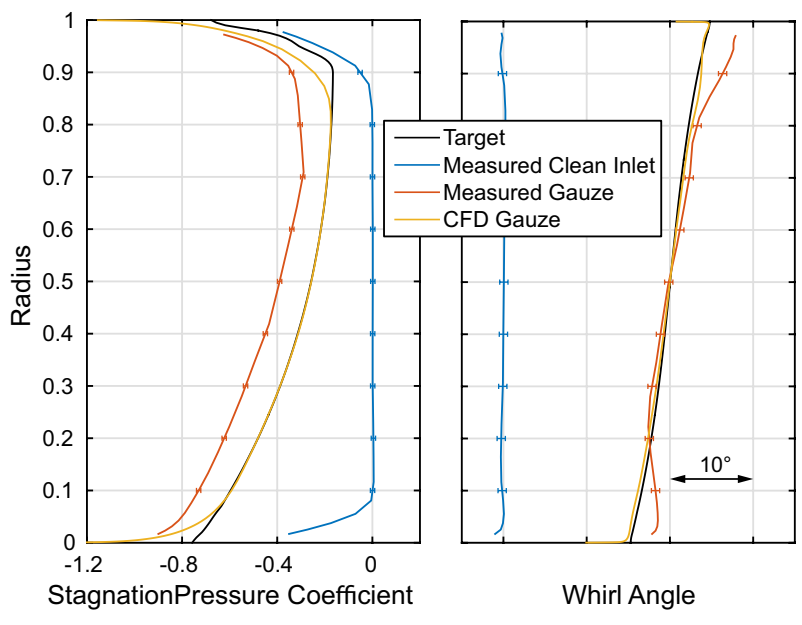

Fig. 10 CFD design and experimental test of core compressor gauze

Increased flow passing through the endwall passages results in increased turning in these regions.

This gauze was more difficult to design than the embedded stage case due to the large absolute variation in stagnation pressure coefficient requiring a large variation in porosity. The limit of minimum trailing edge thickness had to be relaxed at the casing from 0.45 to $0.40 \mathrm{~mm}$ to increase the stagnation pressure in this region. This corresponds to a maximum porosity at the casing of 0.875 . In the first passage off the hub the ratio of maximum thickness to trailing edge thickness also had to be tailored.

\section{Conclusions}

Complete flow conditioning gauzes can be designed to provide any stagnation pressure and whirl angle profile into a rotating rig or wind tunnel experiment. They offer finer control over flow conditioning than existing techniques. The gauzes can be designed reliably using 3D CFD and printed cheaply using commonly available additive manufacture techniques.

Total stagnation pressure losses through the gauzes are relatively high, of the order of $0.3-0.5$ of dynamic head. However, this is not dissimilar to the pressure loss from turbulence generating grids commonly required. It seems likely that most fluid dynamic experiments will adopt a method of flow conditioning like this in the future.

Acknowledgements The author is grateful for the comments, suggestions and encouragement of colleagues at the Whittle Laboratory and Rolls-Royce, most notably Tony Dickens, Chris Hall, Chris Clark, Sam Grimshaw, Malcolm Hillel and Rob Miller. The author would also like to thank Rolls-Royce plc. for permission to publish this work and TURBOSTREAM for the use of their solver.

Open Access This article is distributed under the terms of the Creative Commons Attribution 4.0 International License (http://creativeco mmons.org/licenses/by/4.0/), which permits unrestricted use, distribution, and reproduction in any medium, provided you give appropriate credit to the original author(s) and the source, provide a link to the Creative Commons license, and indicate if changes were made.

\section{References}

Brandvik T, Pullan G (2010) An accelerated 3D Navier Stokes solver for flows in turbomachines. ASME J Turbomach 133(2):021025021025. https://doi.org/10.1115/1.4001192

Guimarães T, Lowe KT, O’Brien WF (2018) Streamvane turbofan inlet swirl distortion generator: mean flow and turbulence structure. $\mathrm{J}$ Propuls Power 34(2):340-353. https://doi.org/10.2514/1.b36422

Gunn E, Hall C (2014) Aerodynamics of boundary layer ingesting fans. In: ASME Turbo Expo 2014: turbine technical conference and exposition, American Society of Mechanical Engineers, pp V01AT01A024-V01AT01A024

Livesey JL, Laws EM (1973) Flow through non-uniform gauze screens. J Fluid Mech 59(4):737-743. https://doi.org/10.1017/s002211207 3001825. https://www.cambridge.org/core/article/flow-throughnonuniform-gauze-screens/ABDF48C288E9FE3DD545CBB4C 8DA5545

McCormick DC, Bennett JC (1994) Vortical and turbulent structure of a lobed mixer free shear layer. AIAA J 32(9):1852-1859. https:// doi.org/10.2514/3.12183

Roach P (1987) The generation of nearly isotropic turbulence by means of grids. Int J Heat Fluid Flow 8(2):82-92

Spalart P, Allmaras S (1992) A one equation turbulence model for aerodynamic flows. AIAA J 1992:94-439

Taylor JV (2015) Three-dimensional mechanisms in compressor flows. $\mathrm{PhD}$ Thesis, Department of Engineering, University of Cambridge

Taylor JV, Miller RJ (2016) Competing three-dimensional mechanisms in compressor flows. J Turbomach 139(2):021009-021010. https ://doi.org/10.1115/1.4034685

Publisher's Note Springer Nature remains neutral with regard to jurisdictional claims in published maps and institutional affiliations. 